\section{Comparison of endothelial cell loss and complications following DMEK with the use of three different graft injectors}

K Droutsas ${ }^{1,2}$, A Lazaridis ${ }^{1,2}$, GD Kymionis ${ }^{2,3}$,

K Chatzistefanou ${ }^{2}$, MM Moschos $^{2}$,

C Koutsandrea ${ }^{2}$ and W Sekundo ${ }^{1}$

\begin{abstract}
Purpose To compare endothelial cell loss and complications following Descemet membrane endothelial keratoplasty (DMEK) with use of three commercially available injectors.

Methods From 179 consecutive DMEK
\end{abstract} procedures, 66 cases with uncomplicated graft preparation and completed 12-month followup were included in this retrospective study. According to the injector used, 3 groups were formed: group 1 (D.O.R.C. injector, $n=16$ ), group 2 (Geuder injector, $n=24$ ), and group 3 (Pasteur laboratory pipette, $n=26$ ).

Endothelial cell density (ECD) and endothelial cell loss were evaluated preoperatively and at 3, 6 and 12 months postoperatively; surgery-related complications were recorded.

Results Donor ECD was $2416 \pm 179$ cells/ $\mathrm{mm}^{2}$ (group 1), $2417 \pm 164$ cells $/ \mathrm{mm}^{2}$ (group 2), and $2478 \pm 234$ cells $/ \mathrm{mm}^{2}$ (group 3 ). At 12 months postoperatively ECD was $1473 \pm 403$ cells $/ \mathrm{mm}^{2}$ (group 1), $1379 \pm 317$ cells $/ \mathrm{mm}^{2}$ (group 2), and $1316 \pm 456 \mathrm{cells} / \mathrm{mm}^{2}$ (group 3) ( $P=0.533$, ANOVA). Endothelial cell loss 12 months postoperatively was $39 \pm 15 \%$ (group 1), $43 \pm 13 \%$ (group 2), and $47 \pm 17 \%$ (group 3) ( $P=0.386$, ANOVA). A single case of iris bleeding and reflux of the graft out of the anterior chamber (both in group 3) was noted. Partial graft detachment occurred in group $1(n=1)$, group $2(n=5)$, and group 3 $(n=7)$ and rebubbling was performed in $6 \%$ (group 1), 17\% (group 2), and 27\% (group 3). Conclusions To our knowledge, this is the first clinical study assessing the safety of three different DMEK injectors. Group 1 (D. O.R.C injector) showed the least and group 3
(Pasteur laboratory pipette) the highest endothelial cell loss without reaching statistical significance at $\mathbf{1 2}$ months after surgery. Therefore, the graft injector may be chosen according to surgeon's preference. Eye (2018) 32, 19-25; doi:10.1038/eye.2017.237; published online 17 November 2017

\section{Introduction}

Endothelial keratoplasty, that is, the transplantation of corneal endothelium, was a major breakthrough of the last decade, allowing faster and better visual rehabilitation while being less invasive than its predecessor, that is, penetrating keratoplasty. ${ }^{1}$ Descemet membrane endothelial keratoplasty (DMEK) is currently the most selective technique of endothelial keratoplasty. In DMEK, only corneal endothelium on its basement membrane, that is, Descemet membrane (DM) is transplanted. ${ }^{2-4}$

The DMEK graft is $15 \mu \mathrm{m}$ thin (depending on donor's age) and forms a roll with the endothelium on the outer surface when submerged in fluid. Thus, direct contact to the graft during preparation and surgery may lead to endothelial cell loss and should be avoided. Endothelial cell loss may occur at all stages of DMEK, that is, during graft preparation, insertion into the anterior chamber unfolding, centering, and appositioning of the donor tissue.

Specifically, insertion of the graft into the anterior chamber involves two passages through the injector opening, (1) aspiration into the injector and (2) insertion into the anterior chamber of the recipient eye. Contact and friction between the graft and the injector wall may lead to variable endothelial cell loss causing
${ }^{1}$ Department of Ophthalmology, Philipps University, Marburg, Germany

${ }^{2}$ First Department of Ophthalmology, National and Kapodistrian University of Athens, Athens, Greece

${ }^{3}$ Jules Gonin Eye Hospital, Faculty of Biology and Medicine, University of Lausanne, Lausanne, Switzerland

Correspondence: K Droutsas, First Department of Ophthalmology, National and Kapodistrian University of Athens, Mesogeion Av. 154, Athens 11527, Greece Tel: +30 6947194809; Fax: +30 2107700504 . E-mail: konstantinos_ droutsas@yahoo.gr

Received: 5 June 2016 Accepted in revised form: 1 August 2017 Published online: 17 November 2017 
reduction of graft survival or even to primary graft failure. ${ }^{5}$

The first device used as a DMEK graft injector was the Pasteur glass pipette, which has a long, narrow ending allowing insertion into the anterior chamber through a $3 \mathrm{~mm}$ incision, without need of sutures. 6,7

Recently, graft injectors specifically designed for DMEK were introduced. The Melles (DMEK injector, D.O.R.C., Zuidland, The Netherlands) and the Szurmann injector (DMEK injector, Geuder, Germany) are also glass-made pipettes with a wide opening for aspiration of the DMEK roll and a narrower opening for insertion into the recipient anterior chamber. Thus, they are designed to avoid endothelial trauma by combining atraumatic aspiration of the graft through the wide opening and insertion into the eye through the narrower opening through a $3.0 \mathrm{~mm}$ cornea incision. In addition, both injectors have a smooth opening surface as opposed to the Pasteur pipette (Figure 1). A recent experimental study showed similar endothelial cell loss among injectors with a size above $0.5 \mathrm{~mm} .^{8}$

Purpose of the present retrospective comparative case series is to analyse and compare the safety of the three aforementioned injector devices used for DMEK surgery, that is, the D.O.R.C. and Geuder injectors and the Pasteur pipette.

\section{Materials and methods}

All medical records and surgery reports of 179 consecutive DMEK surgeries performed between 2010 and 2013 at the Ophthalmology Department of the Philipps University, Marburg, Germany, were reviewed.

Cases with complicated graft preparation, for example, large or multiple tears or excessive manipulations resulting in small or irregular grafts, were excluded.

Then, according to the injector system used, three groups were formed: group 1 (DMEK surgical disposable set, D.O.R.C., Netherlands), group 2 (DMEK implantation cartridge, Geuder, Germany) and group 3 (Pasteur laboratory pipette, VWR, Darmstadt, Germany). In order to reduce a possible learning curve bias related to the use of each injector, the initial 5 cases performed by each surgeon with each of the three injectors (ie, a total of 45 DMEK procedures) were excluded.

Notably, the Pasteur pipette was used exclusively during the first 2 years following the introduction of DMEK at our center (ie, 2010 and 2011), as no other injector was available. Since 2012, however, all three injectors (DORC, Geuder and Pasteur) were used interchangeably. Therefore, in order to reduce possible negative bias related to the learning curve of DMEK surgery, all cases performed in 2010 and 2011 (all with use of the Pasteur pipette) were also excluded.
The following parameters were analysed: recipient age, ocular comorbidities, endothelial cell density (ECD), endothelial cell loss and best-corrected visual acuity (BCVA) before and at 3, 6, and 12 months after DMEK. Surgery-related complications and air reinjection rate (rebubbling) were also recorded.

\section{Protocols of donor tissue preparation and DMEK surgery}

All donor tissue preparations and DMEK surgeries were performed by two surgeons (KD and WS) using the same technique. All procedures were performed following standardized no-touch techniques for graft preparation and DMEK surgery. ${ }^{9,10}$

In short, DM was carefully stripped from the donor stroma and the central 9.0 to $9.5 \mathrm{~mm}$ of the endothelial graft were trephined and submerged in organ culture medium, where the graft spontaneously formed a roll with the endothelial side outwards. The patient was then brought into the operating room and DMEK surgery was performed under subtenon's block. First, the anterior chamber was filled with air and the central $9.0 \mathrm{~mm}$ portion of recipient DM and endothelium was scored and scraped off from the stroma with a reverse Sinskey hook (descemetorhexis). After removing the culture medium and replacing it with balanced salt solution (BSS), the DMEK graft was stained with trypan blue $0.06 \%$, aspirated into the injector and inserted into the anterior chamber of the recipient. Correct orientation of the DMEK graft was assessed by means of the Moutsouris sign; the graft was then unrolled, centered and appositioned onto the recipient stroma by injecting an air bubble underneath it.

The injectors in groups 1 and 2 were used according to the manufacturer's guidelines (http://www.dorc.eu/ literature/dmek_brochure.pdf for group 1 and http:/ / www.geuder.com/dmekinstrumentset for group 2; internet links assessed on 1 June 2016).

In group 3, the Pasteur pipette was filled with BSS, placed coaxially to the rolled DMEK graft and the pressure on the pipette balloon was slowly released in order to create negative pressure high enough to aspirate the DMEK roll.

Graft insertion was performed in all 3 groups in the same manner. While the main incision was being held open with fine toothed forceps, the injector opening was inserted pointing slightly upwards in order to avoid graft insertion into the sulcus. The main port was fashioned wider (ie, around $3.2 \mathrm{~mm}$ ) in group 2 in order to avoid overfilling the anterior chamber due to the larger diameter of the Geuder injector. 

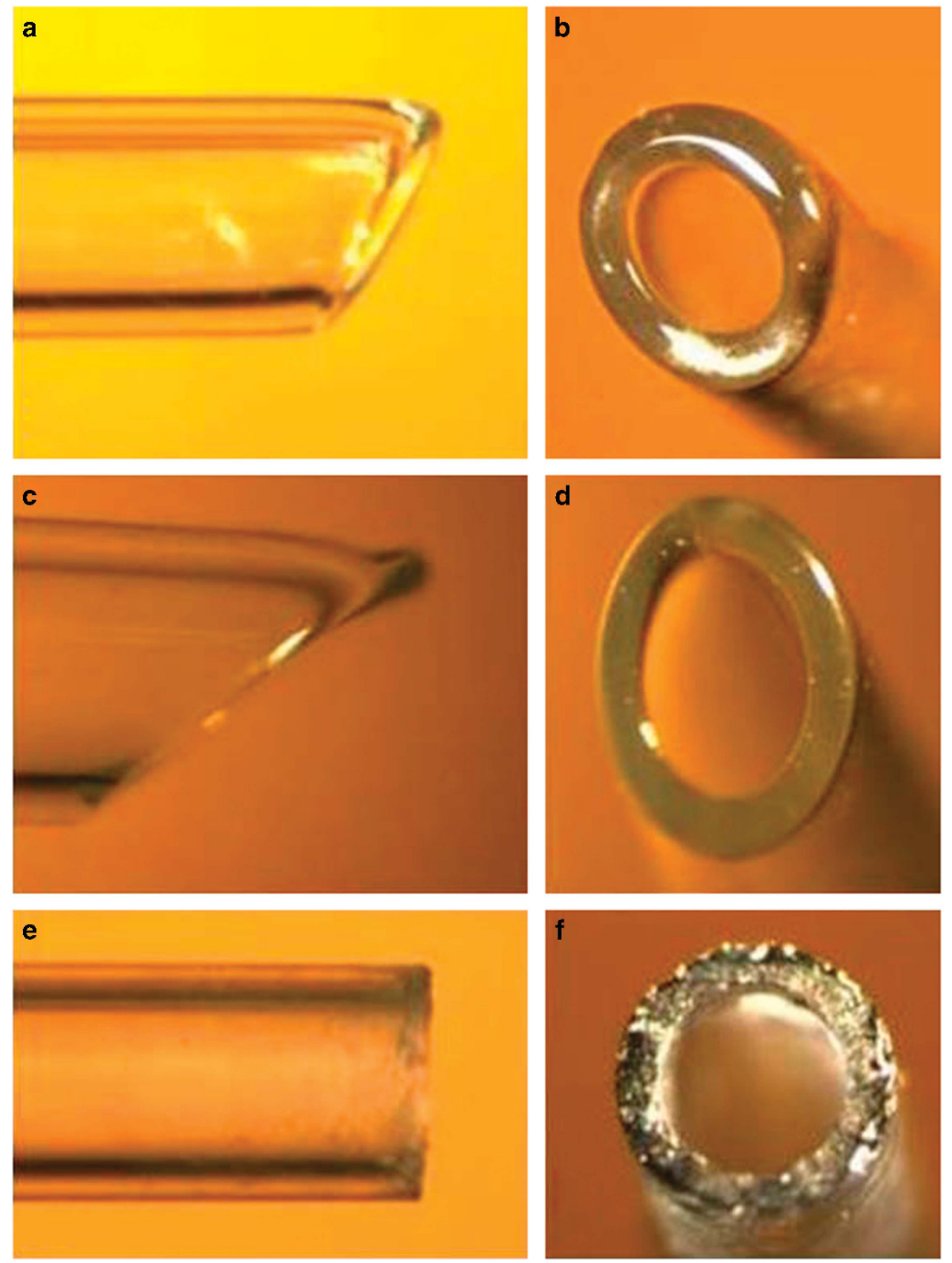

Figure 1 Photo collage depicting lateral (left column) and frontal (right column) views of the three commercially available DMEK assessed in the present paper, that is, the D.O.R.C. injector (a and b), the Geuder injector (c and d) and the Pasteur pipette (e and f). Please note the oblique and smoother opening of the D.O.R.C. and Geuder injectors as opposed to the vertical and rough opening of the Pasteur pipette.

\section{Statistical analysis}

Statistical analysis was performed with SPSS version 17 (SPSS, Inc., Chicago, IL, USA). Descriptive data are presented as central tendency and dispersion. After testing for Normality, comparison of means was conducted either by means of a parametric (one way or repeated measurements ANOVA) or non-parametric test (Kruskal-Wallis test). For nominal data $\chi^{2}$-test was used.

\section{Results}

\section{Demographics}

Recipient demographics are presented in Table 1. A total of 66 DMEK surgeries were included (group 1: $n=16$, group 2: $n=24$ and group 3: $n=26$ ). Recipient age was $68 \pm 11$ years (group 1), $71 \pm 8$ years (group 2 ) and $71 \pm 10$ years (group 3 ). Indications for surgery included corneal endothelial decompensation due to FED $(n=51)$, PBK $(n=11)$, failed DMEK $(n=1)$ and 
Table 1 Demographics

\begin{tabular}{|c|c|c|c|c|}
\hline & Group 1 (D.O.R.C.) & Group 2 (Geuder) & Group 3 (Pasteur) & P-value (statistical test) \\
\hline Eyes & 16 & 24 & 26 & $\chi^{2}=2.545, P=0.28$ \\
\hline Age & $68 \pm 11$ & $71 \pm 8$ & $71 \pm 10$ & \\
\hline \multicolumn{5}{|l|}{ Surgical indications } \\
\hline FED & 13 & 21 & 17 & \\
\hline PBK & 2 & 3 & 6 & \\
\hline Failed DMEK & 1 & 0 & 0 & \\
\hline Failed DSAEK & 0 & 0 & 2 & \\
\hline \multicolumn{5}{|l|}{ Ocular comorbidities } \\
\hline Amblyopia & 1 & 1 & 0 & \\
\hline Epiretinal Membrane & 1 & 0 & 0 & \\
\hline Central Artery Occlussion & 0 & 0 & 1 & \\
\hline \multicolumn{5}{|l|}{ Lens status } \\
\hline pcIOL & 13 & 21 & 23 & \\
\hline acIOL & 0 & 1 & 1 & \\
\hline Phakic & 1 & 0 & 2 & \\
\hline cataract ('Triple DMEK') & 2 & 2 & 0 & \\
\hline \multicolumn{5}{|l|}{ Past surgeries } \\
\hline Vitrectomy & 1 & 1 & 1 & \\
\hline Amniotic membrane & 1 & 0 & 1 & \\
\hline Trabeculectomy & 0 & 0 & 1 & \\
\hline \multicolumn{5}{|l|}{ Intraoperative complications } \\
\hline Graft insertion (iris bleeding) & 0 & 0 & 2 & $\chi^{2}=3.01, P=0.083$ \\
\hline Graft unfolding & 0 & 1 & 0 & $\chi^{2}=1.75, P=0.186$ \\
\hline Graft centration & 3 & 0 & 2 & $\chi^{2}=4.46, P=0.034$ \\
\hline Graft appositioning & 2 & 1 & 1 & $\chi^{2}=1.45, P=0.229$ \\
\hline \multicolumn{5}{|c|}{ Postoperative complications - reinterventions } \\
\hline Partial Graft Detachment & 1 & 5 & 7 & $\chi^{2}=2.17, P=0.141$ \\
\hline Rebubbling & 1 & 4 & 7 & $\chi^{2}=2.38, P=0.122$ \\
\hline Repeat EK & 0 & 0 & 0 & \\
\hline
\end{tabular}

Abbreviations: acIOL, anterior chamber intraocular lens; DMEK, Descemet membrane endothelial keratoplasty; DSAEK, Descemet stripping automated endothelial keratoplasty; EK, endothelial keratoplasty; FED, Fuchs endothelial dystrophy; pcIOL, posterior chamber intraocular lens.

failed DSAEK $(n=2)$. All eyes were pseudophakic with an intraocular lens (IOL) 'in the bag' $(n=57)$, except for 2 eyes with an angle-supported anterior chamber IOL, 3 eyes with a clear crystalline lens (lens was left in situ) and 4 eyes with cataract (treated with phacoemulsification combined with DMEK).

Ocular comorbidities were noted in four cases (amblyopia, $n=2$; epiretinal membrane, $n=1$ and central retinal artery occlusion; $n=1$ ). Previous ocular surgeries included pars-plana-vitrectomy $(n=3)$, amniotic membrane transplantation $(n=2)$ and trabeculectomy $(n=1)$ (Table 1).

Mean diameter of the DMEK graft was $9.1 \pm 0.3 \mathrm{~mm}$ (group 1), $9.0 \pm 0.3 \mathrm{~mm}$ (group 2 and group 3, respectively) ( $P=0.165, \chi^{2}=3.606$, Kruskal-Wallis test) (Table 2).

\section{Endothelial cell density and endothelial cell loss}

ECD before surgery was $2416 \pm 179$ cells $/ \mathrm{mm}^{2}$ (group 1 ), $2417 \pm 164$ cells $/ \mathrm{mm}^{2}$ (group 2), and $2478 \pm 234$ cells $/ \mathrm{mm}^{2}$ (group 3) ( $P=0.489$, One way ANOVA). At 12 months after surgery, mean ECD was $1473 \pm 403$ cells $/ \mathrm{mm}^{2}$ (group 1), $1379 \pm 317$ cells $/ \mathrm{mm}^{2}$ (group 2 ), and $1316 \pm 456$ cells $/ \mathrm{mm}^{2}$ (group 3) $(P=0.533$, one-way ANOVA) (Figure 2 ).

Endothelial cell loss at 3 months after surgery was $27 \pm 20 \%, 32 \pm 16 \%$, and $41 \pm 21 \%$, for groups 1,2 , and 3 , respectively. At 6 months endothelial cell loss was $33 \pm 18 \%$ (group 1), $34 \pm 16 \%$ (group 2), and $44 \pm 21 \%$ (group 3). At 12 months after surgery, endothelial cell loss was $39 \pm 15 \%$ (group 1), $43 \pm 13 \%$ (group 2 ) and $47 \pm 17 \%$ (group 3). A statistically significant ECD reduction was found only at 3 months after surgery between groups 1 and $3(P=0.019$, one-way ANOVA) and between groups 2 and $3(P=0.035$, one-way ANOVA). 
Table 2 Preoperative and postoperative ECD and BCVA data

\begin{tabular}{|c|c|c|c|c|c|}
\hline & $\begin{array}{l}\text { Group } 1 \\
\text { (D.O.R.C.) }\end{array}$ & $\begin{array}{l}\text { Group } 2 \\
\text { (Geuder) }\end{array}$ & $\begin{array}{l}\text { Group } 3 \\
\text { (Pasteur) }\end{array}$ & One way ANOVA & $\begin{array}{c}\text { Repeated measurements } \\
\text { ANOVA }\end{array}$ \\
\hline $\begin{array}{l}\text { Graft } \\
\text { Diameter }\end{array}$ & $9.1 \pm 0.3$ & $9.0 \pm 0.3$ & $9.0 \pm 0.3$ & $\chi^{2}=3.606 P=0.165$ & \\
\hline \multicolumn{6}{|l|}{$E C D$} \\
\hline donor & $2416 \pm 179$ & $2417 \pm 164$ & $2478 \pm 234$ & $F=0.723 P=0.489$ & $F=1.721 P=0.192$ \\
\hline 3 months & $1778 \pm 508$ & $1640 \pm 378$ & $1426 \pm 530$ & $F=2.469 P=0.095$ & \\
\hline 6 months & $1610 \pm 476$ & $1609 \pm 400$ & $1394 \pm 527$ & $F=1.552 P=0.220$ & \\
\hline 12 months & $1473 \pm 403$ & $1379 \pm 317$ & $1316 \pm 456$ & $F=0.638 P=0.533$ & \\
\hline \multicolumn{6}{|l|}{ EC loss } \\
\hline 3 months & $27 \pm 20$ & $32 \pm 16$ & $41 \pm 21$ & $\begin{array}{l}F=3.695 P=0.032(1,2), P=0.675(1,3), \\
P=0.019(2,3), P=0.035\end{array}$ & $F=2.301 P=0.113$ \\
\hline 6 months & $33 \pm 18$ & $34 \pm 16$ & $44 \pm 21$ & $F=2.233 P=0.117$ & \\
\hline 12 months & $39 \pm 15$ & $43 \pm 13$ & $47 \pm 17$ & $F=0.970 P=0.386$ & \\
\hline \multicolumn{6}{|l|}{$B C V A$} \\
\hline preop & $0.75 \pm 0.50$ & $0.68 \pm 0.38$ & $0.55 \pm 0.37$ & $F=0.170, P=0.844$ & $F=0.067 P=0.935$ \\
\hline 3 months & $0.10 \pm 0.17$ & $0.22 \pm 0.26$ & $0.21 \pm 0.35$ & $F=1.088 P=0.343$ & \\
\hline 6 months & $0.08 \pm 0.21$ & $0.12 \pm 0.22$ & $0.18 \pm 0.34$ & $F=1.303 P=0.279$ & \\
\hline 12 months & $0.10 \pm 0.21$ & $0.18 \pm 0.30$ & $0.27 \pm 0.20$ & $F=0.376 P=0.688$ & \\
\hline
\end{tabular}

Abbreviations: BCVA, best-corrected visual acuity; EC loss, endothelial cell loss; ECD, endothelial cell density; logMAR, logarithm of minimum angle of resolution.

\section{Intraoperative complications}

Regarding complications during graft insertion, one case of iris bleeding and one case of graft reflux out of the eye were noted in group 3. Iris bleeding was probably caused by centripetal pushing of the iris by the injector tip during insertion. Reflux of the graft was attributed to obstruction of the main port by the large injector tip of the Geuder injector, leading to overinflation of the chamber and reflux of BSS along with the graft during retraction of the injector tip from the main port. The graft was carefully grasped with McPherson forceps, placed into BSS, stained with trypan blue $0.06 \%$, reinserted into the anterior chamber and surgery was completed without further complications (Table 1).

No complications were recorded with regard to unfolding manoeuvres of the graft. Nevertheless, difficulty with graft centration was encountered in group $1(n=3)$ and group $3(n=2)$. Here the grafts were centered by pressing them onto the iris and pulling them to position with a $30 \mathrm{G}$ cannula (Table 1).

Finally, suboptimal appositioning with peripheral inward folds of the DMEK graft at the end of surgery was documented in group $1(n=2)$, group $2(n=1)$, and group 3 $(n=1)$ (Table 1).

\section{Graft detachment and rebubbling}

A partial graft detachment was documented in group 1 $(n=1)$, group $2(n=5)$, and group $3(n=7)(P=0.141$,

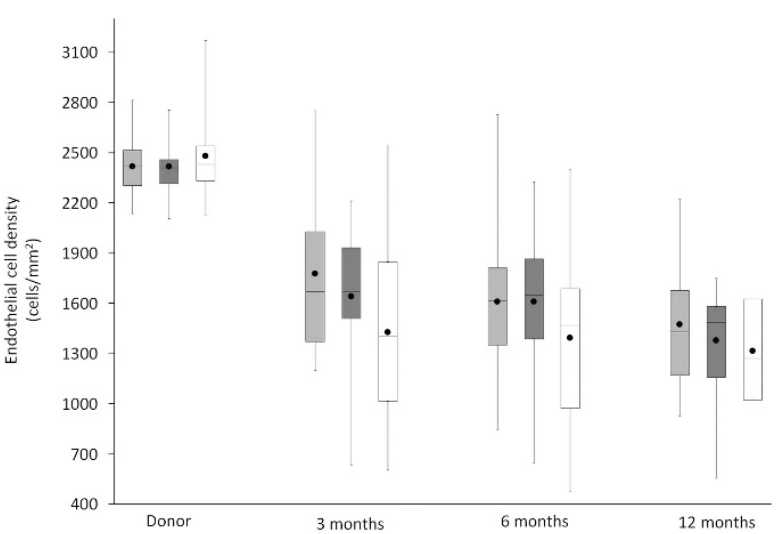

Figure 2 Box plots depicting the course of endothelial cell density (ECD) ( $y$ axis) over time ( $x$ axis) after DMEK with the use of three different injectors (group 1: D.O.R.C. injector/blue, group 2: Geuder injector/red, group 3: Pasteur pipette/white). Notably, group 3 shows the highest donor ECD and the lowest ECD at 12 months after surgery (not statistically significant). A full color version of this figure is available at the Eye journal online.

$\chi^{2}=2.17, \chi^{2}$-test). Rebubbling was performed within the first postoperative month in cases with central detachment interfering with vision or progressive peripheral detachment larger than $1 / 3$ of the graft area. This was documented in $6 \%$ (group 1), $17 \%$ (group 2), and $27 \%$ (group 3) $\left(P=0.122, \chi^{2}=2.38, \chi^{2}\right.$-test). All rebubbling procedures led to satisfactory clearing of the central cornea (Table 1). 


\section{Best corrected visual acuity}

Preoperative $\log$ MAR was $0.75 \pm 0.50$ (group 1$)$, $0.68 \pm 0.38$ (group 2), and $0.55 \pm 0.37$ (group 3) $(P=0.844$, one-way ANOVA). At 12 months, $\operatorname{logMAR}$ was $0.1 \pm 0.21$ (group 1), $0.18 \pm 0.3$ (group 2), and $0.27 \pm 0.20$ (group 3) $(P=0.688$, one-way ANOVA) (Figure 3$)$. Also here, no significant difference in logMAR was found among the three groups at any time point $(P=0.935$, repeated measurements ANOVA) (Table 2).

\section{Discussion}

To our knowledge this is the first clinical comparison of three commercially available DMEK injectors in terms of endothelial cell loss, intra-, and postoperative complications.

Group 1 (D.O.R.C. injector) demonstrated the lowest, whereas group 3 (Pasteur pipette) the highest endothelial cell loss among the 3 groups at each follow-up exam (Table 2). These differences were statistically significant only in the 3rd postoperative month.

This finding may be explained by inherent disadvantages of the Pasteur pipette when compared to the other two injectors which are specifically designed for DMEK: (1) with the Pasteur pipette, the DMEK roll is aspirated through a narrow opening causing friction and cell loss during passage of the graft and (2) the vertical opening cut of the Pasteur pipette may cause more trauma to the endothelial monolayer during graft aspiration as opposed to the smooth, curved edge profile of the D.O.R.C. and Geuder injectors (Figure 1). Hence, the use of a Pasteur pipette may account for slight differences in reported endothelial cell loss after DMEK. ${ }^{6,7}$

The rate of injector-related complications and reinterventions was similar in all groups. Nevertheless, the vertical opening cut of the Pasteur pipette could account for iris bleeding due to centripetal dragging of iris tissue during insertion of the injector into the anterior chamber. In contrast, the other two injectors glide on iris tissue as they have a smooth and oblique opening cut (Figure 1).

Another injector-related complication was the reflux of the graft out of the caused by overinflation of the anterior chamber during graft insertion due to obstruction of the main incision by the injector. Thus, as the injector is retracted, BSS along with the graft may flow out of the eye. This was observed in one case (group 2) and occurred probably due to the wide opening of the Geuder injector; either a larger main incision or simultaneous opening of a side port during graft insertion could be helpful in order to avoid anterior chamber overinflation.

Finally, one would expect to observe higher trauma with use of narrower injectors. However, a recent in vitro comparison of glass injectors with different diameters

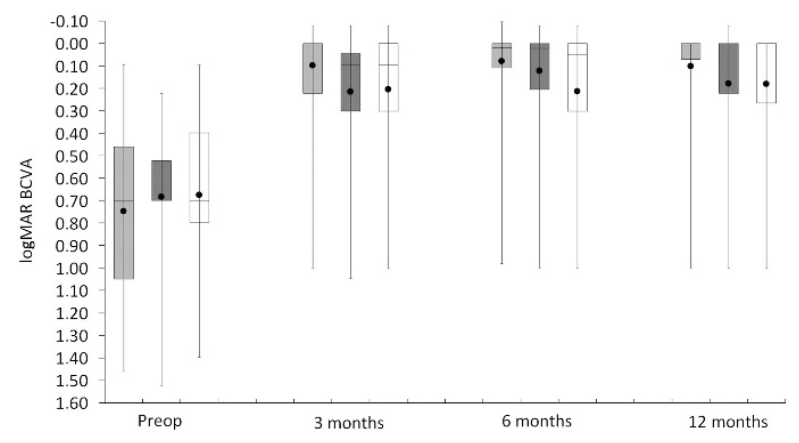

Figure 3 Box plots depicting best corrected visual acuity (BCVA, logMAR values) ( $y$ axis) over time ( $x$ axis) after DMEK with the use of three different injectors (group 1: D.O.R.C. injector/blue, group 2: Geuder injector/red, group 3: Pasteur pipette/white). BCVA increases continuously with the steepest increase located between 1 week and 1 month after DMEK. Although group 1 demonstrates slightly higher BCVA, no statistically significant levels were reached. A full color version of this figure is available at the Eye journal online.

\section{$(0.5,0.9$, and $1.4 \mathrm{~mm})$ did not reveal statistically} significant differences in cell viability. ${ }^{8}$ Similarly, only marginal differences in endothelial cell loss among the three commercially available injectors with similar lumen diameters were found in the present report (Table 2). Our clinical study, however, evaluates the real-time behaviour of endothelial cells as opposed to in-vitro studies which may document the immediate damage, but do not shed any light on mid-term cell survival.

\section{Conclusion}

All three injector systems demonstrated similar outcomes in terms of surgery-related complications and endothelial cell loss. Patients in group 1 (D.O.R.C injector) showed less endothelial cell loss compared to group 2 (Geuder injector), and group 3 (Pasteur laboratory pipette), reaching statistically significant levels at 3 months after surgery.

\section{Summary}

\section{What was known before}

- Contact and friction occurring between the graft and the injector wall may lead to variable endothelial cell loss causing reduction of graft survival or even to primary graft failure. A recent ex vivo experimental study showed similar endothelial cell loss among injectors with a size opening $>0.5 \mathrm{~mm}$.

What this study adds

- This is the first study that compares and evaluates the safety of 3 commercially available injector devices used for DMEK surgery, that is, the D.O.R.C. and Geuder injectors and the Pasteur pipette. All three injector systems demonstrated similar outcomes in terms of surgery-related complications and endothelial cell loss. 


\section{Conflict of interest}

The authors declare no conflict of interest.

\section{Acknowledgements}

We confirm that we had full access to all the data in the study and take responsibility for the integrity of the data and the accuracy of the data analysis. We thank Dr Socrates Sklavos, $\mathrm{PhD}$, for his support with statistics.

\section{Author contributions}

Design and conduct of the study (KD and AL); collection, management, analysis, and interpretation of the data $(\mathrm{KD}$, $\mathrm{AL}, \mathrm{GK}, \mathrm{MM}, \mathrm{KC}, \mathrm{CK}$, and WS); preparation of the manuscript (KD and $\mathrm{AL}$ ); review, or approval of the manuscript (GK, MM, KC, CK, and WS).

\section{References}

1 Melles GR. Posterior lamellar keratoplasty: DLEK to DSEK to DMEK. Cornea 2006; 25(8): 879-881.

2 Melles GR, Ong TS, Ververs B, van der Wees J. Descemet membrane endothelial keratoplasty (DMEK). Cornea 2006; 25(8): 987-990.

3 Ham L, Dapena I, van Luijk C, van der Wees J, Melles GR. Descemet membrane endothelial keratoplasty (DMEK) for
Fuchs endothelial dystrophy: review of the first 50 consecutive cases. Eye (Lond) 2009; 23(10): 1990-1998.

4 Droutsas K, Ham L, Dapena I, Geerling G, Oellerich S, Melles G. Visual acuity following Descemet-membrane endothelial keratoplasty (DMEK): first 100 cases operated on for Fuchs endothelial dystrophy. Klin Monbl Augenheilkd 2010; 227(6): 467-477.

5 Ham L, van der Wees J, Melles GR. Causes of primary donor failure in descemet membrane endothelial keratoplasty. Am J Ophthalmol 2008; 145(4): 639-644.

6 Droutsas K, Giallouros E, Melles GR, Chatzistefanou K, Sekundo W. Descemet membrane endothelial keratoplasty: learning curve of a single surgeon. Cornea 2013; 32(8): 1075-1079.

7 Monnereau C, Quilendrino R, Dapena I, Liarakos VS, Alfonso JF, Arnalich-Montiel F et al. Multicenter study of descemet membrane endothelial keratoplasty: first case series of 18 surgeons. JAMA Ophthalmol 2014; 132(10): 1192-1198.

8 Yoeruek E, Bartz-Schmidt KU, Hofmann J. Impact of the radius of the injector system on the cell viability in descemet membrane endothelial keratoplasty: an ex vivo experimental study. Acta Ophthalmol 2016; 94(1): e1-e5.

9 Lie JT, Birbal R, Ham L, van der Wees J, Melles GR. Donor tissue preparation for Descemet membrane endothelial keratoplasty. J Cataract Refract Surg 2008; 34(9): 1578-1583.

10 Dapena I, Moutsouris K, Droutsas K, Ham L, van Dijk K, Melles GR. Standardized 'no-touch' technique for descemet membrane endothelial keratoplasty. Arch Ophthalmol 2011; 129(1): 88-94. 